\title{
Original
}

\section{Antibacterial Activity and Biocompatibility of Nanoporous Titanium Doped with Silver Nanoparticles and Coated with N-Acetyl Cysteine}

\author{
Honghao Zhang ${ }^{1,2)}$, Mai Hatoko ${ }^{2)}$, Derong Yin $^{2)}$, Yuanyuan Yang ${ }^{2)}$, Yuhao Zeng ${ }^{2)}$, Satoshi Komasa ${ }^{2)}$, Tetsuji Kusumoto ${ }^{3)}$, \\ Hiroshi Nishizaki ${ }^{3)}$, Hideo Shimizu ${ }^{4)}$ Wanghong Zhao $^{1)}$ and Joji Okazaki ${ }^{2)}$
}

\author{
${ }^{1)}$ Department of Stomatology, Nanfang Hospital, Southern Medical University, Guangzhou, China \\ ${ }^{2)}$ Department of Removable Prosthodontics and Occlusion, Osaka Dental University, Osaka, Japan \\ ${ }^{3}$ Department of Oral Health Engineering, Faculty of Health Science, Osaka Dental University, Osaka, Japan \\ ${ }^{4)}$ Osaka University, Osaka, Japan \\ (Accepted for publication, June 26, 2018)
}

\begin{abstract}
Alkali-treated titanium with nanonetwork structure (TNS) is strongly osteogenic but not antibacterial in nature. In contrast, titanium doped with silver nanoparticles (Ag-NPs) possesses excellent and long-term antibacterial activity, but induces significant levels of intracellular reactive oxygen species that may cause cytotoxicity. We attempted to enhance the antibacterial activity of TNS by doping with Ag-NPs and prevent intracellular reactive oxygen species formation by coating with the antioxidant $\mathrm{N}$-acetyl cysteine. Importantly, TNS doped with Ag-NPs and coated with $\mathrm{N}$-acetyl cysteine has similar antibacterial activity against Staphylococcus aureus as TNS doped with only Ag-NPs, and inhibited bacterial attachment, proliferation, and biofilm formation by Actinomyces oris. However, TNS doped with Ag was significantly cytotoxic for rat bone marrow mesenchymal stem cells, whereas TNS doped with Ag and coated with N-acetyl cysteine supported cell viability and differentiation to a certain extent by suppressing overproduction of intracellular reactive oxygen species. Collectively, our results suggest that application of N-acetyl cysteine enhances the biocompatibility of TNS doped with Ag-NPs without compromising antibacterial activity, yielding a material with clinical potential.
\end{abstract}

Key words: Antibacterial activity, Biocompatibility, N-acetyl cysteine, Reactive oxygen species, Silver nanoparticles

\section{Introduction}

Owing to increase in demand, the biological performance and osteoinductivity of orthopaedic and dental implants have been improved through various innovations ${ }^{1)}$. Unfortunately, the frequency of implant-related infections is also increasing, resulting in patient illness and increased medical expense ${ }^{2)}$. Recently, implants doped with one or more metal nanoparticles, e.g., $\mathrm{Ag}, \mathrm{Zn}$, and $\mathrm{Mg}$, were shown to possess robust and stable antibacterial activity ${ }^{3-6)}$. In addition, metal nanoparticles are less likely to elicit bacterial tolerance than antibiotics, while slow and sustained metal release guarantees long-term antibacterial effects.

In particular, silver nanoparticles are considered excellent implant coatings because of their activity against a broad spectrum of microbes $^{7,8)}$. Specifically, mobile Ag nanoparticles kill bacteria by increasing cell membrane permeability and disrupting metabolism ${ }^{7-9)}$, whereas deposited Ag nanoparticles may kill bacteria via other mechanisms because of additional interactions between base materials and bacteria. For example, silver nanoparticles doped into implants may elicit overproduction of reactive oxygen species (ROS), which are reactive molecules and free radicals generated from molecular oxygen typically during mitochondrial electron transport and aerobic respiration or during oxidoreductase- and metal-catalysed oxidation. While these species may disrupt the oxidant/antioxidant balance in bacteria, damage bacterial organelles,

Correspondence to: Dr. Wanghong Zhao, Department of Stomatology, Nanfang Hospital, Southern Medical University, 1838 Guangzhou Avenue North, Guangzhou 510515, China; Tel: +86-020-62787687; Fax: +86-020-62787679; E-mail: zhaowh@smu.edu.cn and impede normal bacterial function, they may also induce imbalance in oxidative stress reactions and cytotoxicity in mammalian cells and thereby inhibit cell proliferation ${ }^{10)}$. Nevertheless, the effect of implant $\mathrm{Ag}$ nanoparticles on ROS in mammalian cells is still not completely understood.

$\mathrm{N}$-acetyl cysteine (NAC) is a small amino acid derivative used not only to treat respiratory diseases but also to promote osteogenic differentiation. In addition, NAC protects mammalian cells by scavenging free radicals or other harmful molecules via a reactive sulfhydryl group ( $-\mathrm{SH}$ ), or as a precursor to reduced glutathione, which maintains the intracellular redox balance. Intriguingly, NAC also possesses antibacterial properties ${ }^{11-16)}$. For example, it robustly and significantly enhances the antibacterial activity of $\mathrm{Ag}$ nanoparticles ${ }^{17}$, and may therefore reduce the required dose of nanoparticles. Therefore, we hypothesized that NAC may not only reduce the cytotoxicity of Ag-doped implants, but may also inhibit bacterial attachment and biofilm formation. To test this hypothesis, it is necessary to investigate (1) whether NAC protects host cells and bacteria from ROS generated by silver nanoparticles, and (2) whether NAC restores cell viability and differentiation.

Previously, we demonstrated that strong alkali treatment of titanium generates a basic, hydrophilic sodium titanate layer with nanonetwork structure. These materials exhibit enhanced osteogenic activity in vitro and in vivo, are fabricated in controllable conditions without heating and hence consume minimal energy, are environmentally friendly, and less expensive $^{18,19)}$. However, these materials are susceptible to bacterial attachment and biofilm formation, which may easily result in infection 
post-implantation or in long-term peri-implantitis ${ }^{20)}$. In this study, titanium with nanonetwork structure (TNS) was further modified with Ag nanoparticles by immersing in $\mathrm{AgNO}_{3}$, followed by ultraviolet chemical reduction to enhance its antibacterial activity. In addition, the material was coated with NAC through layer-by-layer assembly method to reduce intracellular ROS levels and further enhance the antibacterial effect. The antibacterial activity of this material was tested against Staphylococcus aureus and Actinomyces oris. S. aureus is the most common pathogen in peri-implantation infections, especially suppurative implant $\operatorname{sites}^{21)}$, however its ability to adhere and form biofilms is weaker than those of early colonizers such as $A$. oris ${ }^{22)}$, which is prevalent in shallow, deep, and very deep periodontitis ${ }^{23)}$. In addition, $A$. oris adheres and forms biofilms more efficiently than other common pathogens in peri-implantitis. Thus, anti-attachment and anti-biofilm activity against A. oris may also prevent adherence of common agents of peri-implantitis, including $S$. aureus and $P$. gingivalis, which can then be easily eliminated by the immune system as planktonic cells. Meanwhile, rat bone marrow mesenchymal stem cells (rBMMSCs) were also utilized in this study for analyses of biocompatibility of this material, as these cells are at the forefront of the osseointegration process. We anticipate that our results will provide a rationale for applying NAC and $\mathrm{Ag}$ on orthopedic and dental implants.

\section{Materials and Methods \\ Materials, experiment reagents and bacteria purchase}

Medically pure titanium was purchased from Engineering Test Service Ltd. (Osaka, Japan). Ethanol, methanol, acetone, and $\mathrm{AgNO}_{3}$ were purchased from Nacalai Tesque Inc. (Kyoto, Japan). NAC was obtained from Sigma-Aldrich Ltd. (St. Louis, MO, USA), whereas trypticase soy agar and broth were procured from Becton, Dickinson, and Company. $S$. aureus (ATCC 12600) and A. oris (MG-1) were purchased from the Department of Bacteriology, Osaka Dental University.

\section{Fabrication of titanium discs}

Discs of grade 2 commercially pure titanium (diameter $12 \mathrm{~mm}$, thickness $1 \mathrm{~mm}$ ) were successively polished to $800 \#$ using $\mathrm{SiC}$ abrasive paper. The discs were then ultrasonically cleaned in acetone, ethanol, and deionized water for $10 \mathrm{~min}$ each, and air-dried at room temperature overnight. To generate surface nanonetwork structures, cleaned titanium discs were immersed in $10 \mathrm{M} \mathrm{NaOH}$ at $30^{\circ} \mathrm{C}$ for $24 \mathrm{~h}$, rinsed several times with ion-exchanged water until the conductivity of the solution dropped below $5 \mu \mathrm{S} / \mathrm{cm}^{3}$, and air-dried at room temperature overnight. Subsequently, some alkali-treated discs were immersed in $0.1 \mathrm{M} \mathrm{AgNO}_{3}$ at $40^{\circ} \mathrm{C}$ for $3 \mathrm{~h}$, lightly washed in deionized water, air-dried, irradiated for $15 \mathrm{~min}$ at $\lambda=254 \mathrm{~nm}$ and $100 \mathrm{~mW} / \mathrm{cm}^{2}$, and ultrasonically washed in ion-exchanged water for $1 \mathrm{~min}$. All discs were then sterilized in $75 \%$ ethanol for $15 \mathrm{~min}$ and stored in sterile conditions. For coating, Agdoped discs were immersed in $2 \mathrm{mM}$ and $5 \mathrm{mM}$ NAC and air-dried on a clean bench in several cycles until appropriate amounts of NAC were deposited. All titanium discs were divided into the following four groups: TNS (titanium with nanonetwork structure), TNS-Ag (Ag-doped titanium with nanonetwork structures), Ag-NAC-2 (Ag-doped titanium with nanonetwork structures coated with 2 mM NAC) and Ag-NAC-5 (Ag-doped titanium with nanonetwork structures coated with $5 \mathrm{mM}$ NAC).

\section{Surface characterization}

The discs were imaged using an S-4800 scanning electron microscope (Hitachi Ltd., Tokyo, Japan) to assess surface topography. Sam- ples were also analyzed using the Kratos Axis Ultra X-ray photoelectron spectroscope (Shimadzu Ltd., Kyoto, Japan) to assess the chemical composition. Contact angles were measured at room temperature on a DSA 10 Mk2 drop shape analysis system (KRÜSS Ltd., Hamburg, Germany) using $2 \mu 1$ double distilled $\mathrm{H}_{2} \mathrm{O}$.

\section{Antibacterial activity}

For antibacterial rate, S. aureus (ATCC 12600) and A.oris (MG-1) were streaked on trypticase soy agar and incubated at $37^{\circ} \mathrm{C}$ for $24 \mathrm{~h} \mathrm{un-}$ der aerobic conditions. A single colony was subsequently picked and cultivated overnight in $2 \mathrm{ml}$ trypticase soy broth. This seed culture was then diluted in fresh trypticase soy broth to optical density 0.1 at $600 \mathrm{~nm}$ that measured on a colourwave colorimeter (Colourwave CO7500 colorimeter, Funakoshi Ltd., Tokyo, Japan), of which $1 \mathrm{ml}$ was seeded on discs in standard 24-well polystyrene culture plates, and incubated aerobically at $37^{\circ} \mathrm{C}$ for $24 \mathrm{~h}$. After incubation, the bacteria adhered on the discs were collected ultrasonically in $5 \mathrm{ml}$ fresh broth, incubated aerobically at $37^{\circ} \mathrm{C}$ for $24 \mathrm{~h}$, and the optical density at $600 \mathrm{~nm}$ was measured on the same colorimeter. Antibacterial activity was calculated as $\%=$ (A-B) $/ \mathrm{A} \times 100 \%$, where $\mathrm{A}$ is the optical density at $600 \mathrm{~nm}$ of bacteria attached on TNS, and B is the optical density at $600 \mathrm{~nm}$ of bacteria attached on TNS-Ag, Ag-NAC-2 and Ag-NAC-5.

For bacterial formation and attachment, seed cultures of $A$. oris (MG-1) were obtained as described, diluted to optical density 0.1 and 1.0 at $600 \mathrm{~nm}$ to assess biofilm formation and attachment, respectively, seeded on discs as described, and incubated for 6-24 h and 1-6 h, respectively. To assess bacterial attachment, the bacterial suspension was removed after incubation, and the discs were gently transferred to a fresh 24-well polystyrene culture plate, rinsed with $1 \mathrm{ml}$ sterile phosphate-buffered saline (PBS) to dislodge and remove unattached bacteria, and stained for 15 min with Live/Dead ${ }^{\circledR}$ BacLight ${ }^{\mathrm{TM}}$ bacterial viability kit (L7012) following the manufacturer's instructions. Excess dye was then removed, and the discs were immediately photographed on a cover glass under an LSM700 confocal laser-scanning microscope (Carl Zeiss Ltd., Oberkochen, Germany). To assess biofilm formation, the discs were rinsed with PBS, incubated for $20 \mathrm{~min}$ at room temperature with 2 $\mathrm{ml} 0.05 \% \mathrm{w} / \mathrm{v}$ crystal violet dye, rinsed thrice with PBS to remove residual dye, transferred to a new 12-well plate, and destained for $20 \mathrm{~min}$ at room temperature with rotary shaking in $1 \mathrm{ml} \mathrm{95 \%} \mathrm{ethanol.} \mathrm{After}$ destaining, $100 \mu 1$ ethanol was transferred to each well and the absorbance at $595 \mathrm{~nm}$ was determined on a SpectraMax M5 96-well microplate reader (Molecular Devices Ltd., Sunnyvale, CA, USA).

\section{Biocompatibility}

rBMMSCs were harvested from the femurs of 8-week-old Sprague-Dawley rats (SHIMIZU Laboratory Supplies Co., Kyoto, Japan), resuspended in Eagle's minimum essential medium containing $10 \%$ fetal bovine serum and antibiotic-antimycotic mix (all from Nacalai Tesque Inc., Kyoto, Japan), and cultured at $37^{\circ} \mathrm{C}$ in a humidified atmosphere with $5 \% \mathrm{CO}_{2}$. The medium was replaced after every $3 \mathrm{~d}$. At $\sim 80 \%$ confluency, the cells were treated with $0.5 \mathrm{~g} / 1$ trypsin and 0.53 mmol/1 EDTA (Nacalai Tesque Inc., Kyoto, Japan), and seeded in 24well plates containing titanium discs at $4 \times 10^{4}$ cells/well. The culture medium was subsequently replaced after every $3 \mathrm{~d}$.

Cell viability was analyzed at 1,4 , and $7 \mathrm{~d}$ using CellTiter-Blue ${ }^{\circledR}$ cell viability assay (Promega Co., Madison, WI, USA), according to the manufacturer's protocol. Briefly, the samples were washed with PBS, treated for $1 \mathrm{~h}$ at $37^{\circ} \mathrm{C}$ with $300 \mu \mathrm{l}$ diluted CellTiter-Blue ${ }^{\circledR}$ reagent (50 $\mu 1$ CellTiter-Blue ${ }^{\circledR}$ reagent diluted in $250 \mu \mathrm{l}$ PBS), of which $100 \mu 1$ was 


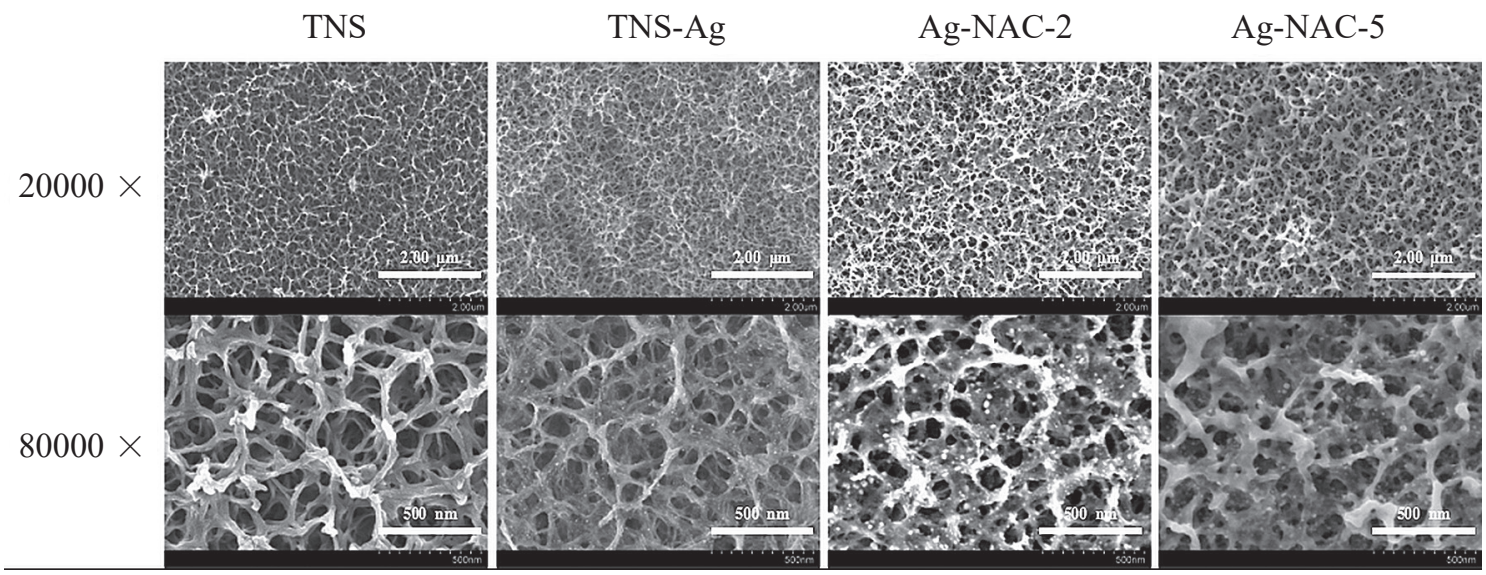

Figure 1. Scanning electron micrographs of titanium network structures (TNS), Ag-doped titanium with nanonetwork structures (TNS-Ag), Ag-doped titanium with nanonetwork structures coated with $2 \mathrm{mM}(\mathrm{Ag}-\mathrm{NAC}-2)$ and $5 \mathrm{mM} \mathrm{N}$-acetyl cysteine (Ag-NAC-5). Scale bar $=2.00 \mu \mathrm{m}$ for $20000 \times$ and scale bar $=500 \mathrm{~nm}$ for $80000 \times$.
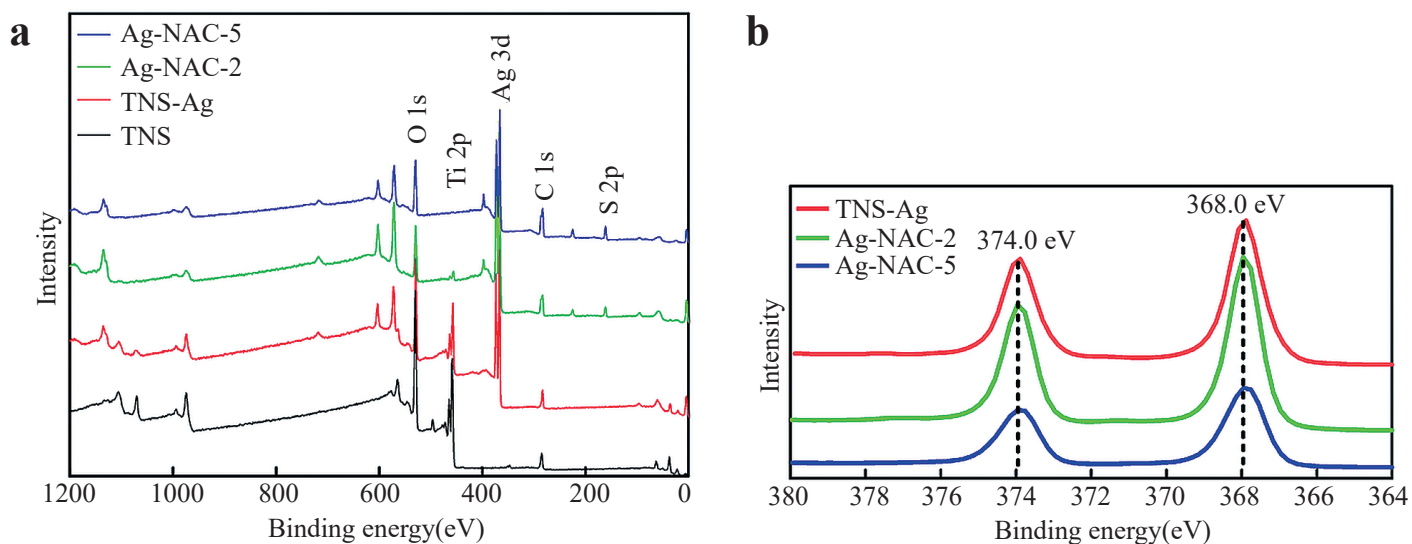

Figure 2. Spectra of various titanium nanostructures (TNS). (a) Full and (b) Ag 3d spectra of TNS, Ag-doped titanium with nanonetwork structures (TNS-Ag), and Ag-doped titanium coated with $2 \mathrm{mM}$ (Ag-NAC-2) and $5 \mathrm{mM} \mathrm{N}$-acetyl cysteine (Ag-NAC-5). Verticle axis: intensity of the peaks; horizontal axis: binding energy of the peaks.

transferred to a new 96-well tissue culture plate and assayed for fluorescence at 560/590 nm using a SpectraMax M5 microplate reader (Molecular Devices Ltd., San Jose, CA, USA).

To evaluate alkaline phosphatase activity, $4 \times 10^{4}$ cells were seeded on discs immersed in $\alpha$-MEM (Nacalai Tesque, Kyoto, Japan) containing $10 \%$ fetal bovine serum, antibiotic-antimycotic mix, $10 \mathrm{mM} \beta$-glycerophosphate (Wako Pure Chemical Industries, Osaka, Japan), ascorbic acid (Nacalai Tesque Inc., Kyoto, Japan), and $10 \mathrm{nM}$ dexamethasone (Nacalai Tesque Inc., Kyoto, Japan). The differentiation medium was changed after every $3 \mathrm{~d}$. After 7 or $14 \mathrm{~d}$, the samples were washed with PBS and lysed with $300 \mu 1$ of $0.2 \%$ Triton X-100. The resulting lysates were transferred to microcentrifuge tubes and assayed using enzyme-linked immunosorbent assay (Sigma-Aldrich Ltd., St. Louis, MO, USA) following the manufacturer's protocol and $200 \mu$ p-nitrophenyl phosphate as the substrate. The reaction was terminated by adding $50 \mu 1$ $3 \mathrm{~N} \mathrm{NaOH}$, and p-nitrophenol release was quantified at $405 \mathrm{~nm}$ using SpectraMax ${ }^{\circledR}$ M5 (Molecular Devices Ltd., San Jose, CA, USA). Alkaline phosphatase activity was normalized to DNA content, which was determined using PicoGreen dsDNA assay kit (Thermo Fisher Life Technologies Ltd., Tokyo, Japan).

To assay intracellular ROS, rBMMSCs were incubated on titanium discs for $3 \mathrm{~d}$. The medium was then replaced with $1 \mathrm{ml}$ fresh medium containing $5 \mu \mathrm{M}$ CellROX ${ }^{\circledR}$ oxidative stress reagents (C10422, Thermo
Fisher Life Technologies Ltd., Tokyo, Japan), and the samples were incubated at $37^{\circ} \mathrm{C}$ for $15 \mathrm{~min}$, washed thrice with PBS, fixed in $4 \%$ paraformaldehyde for $15 \mathrm{~min}$ at room temperature, and imaged using an LSM700 confocal laser-scanning microscope (Carl Zeiss Ltd., Oberkochen, Germany). The fluorescence intensity of the images were analysed by the embedded software of the confocal laser-scanning microscope.

\section{Statistical analysis}

Surface characteristics, bacterial experiments, and cell experiments were each conducted in triplicate. All quantitative results were expressed as means \pm standard deviations. Results were evaluated by oneway analysis of variance (ANOVA) and Bonferroni's post hoc test using SPSS 20.0 software (IBM Co., Armonk, NY, USA); $p<0.05$ was considered statistically significant.

\section{Surface characteristics}

\section{Results}

As shown in Fig. 1, nanoscale reticular network structures were formed on titanium surfaces immersed in strong alkali for $24 \mathrm{~h}$. These structures were slightly damaged after subsequent immersion in $\mathrm{AgNO}_{3}$ and ultraviolet chemical reduction, although small white particles, probably $\mathrm{Ag}$ and $10 \sim 40 \mathrm{~nm}$ in size, were abundantly and uniformly deposit- 


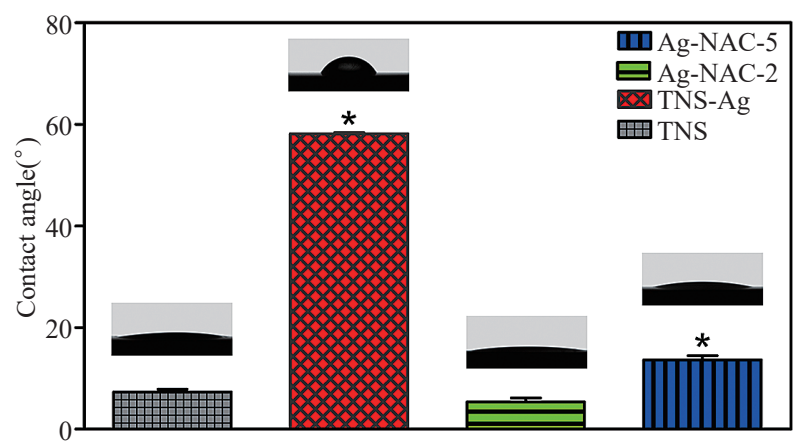

Figure 3. Contact angles of various titanium with nanonetwork structures (TNS). Histogram shows the contact angles of TNS, Ag-doped titanium with nanonetwork structures (TNS-Ag), and Ag-doped titanium coated with $2 \mathrm{mM}$ (Ag-NAC-2) and $5 \mathrm{mM} \mathrm{N}$-acetyl cysteine (Ag-NAC-5). ${ }^{*} p<$ 0.05 vs. titanium with nanonetwork structures (TNS). Insets are representative examples of water droplets.

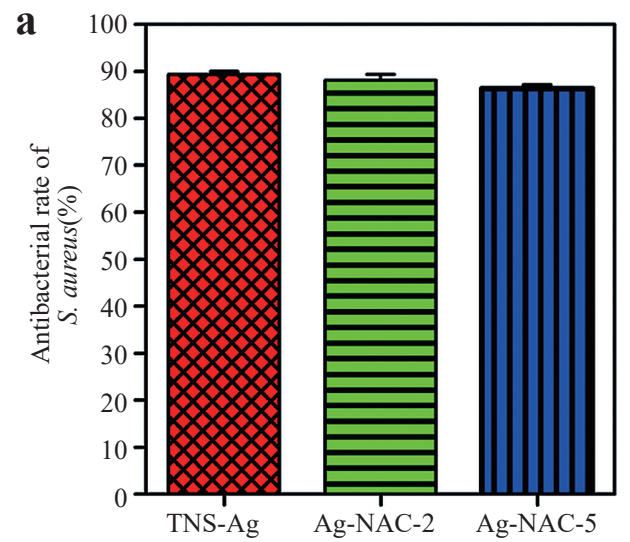

ed. Compared to TNS and TNS-Ag, certain small molecules were deposited on the Ag-NAC sample surface. These molecules attached to the reticular nanoporous structure on the surface but did not completely cover the surface such that the structures were still clear and distinct. In addition, Ag-NAC-5 showed more attached molecules than Ag-NAC-2, and the small white particles were observed on both Ag-NAC-2 and AgNAC-5 surfaces.

On photoelectron spectroscopy (Fig. 2), Ag 3d doublet peaks were observed in TNS-Ag, Ag-NAC-2, and Ag-NAC-5, but not in TNS. Theses peaks were observed at $374.0 \mathrm{eV}(\mathrm{Ag} 3 \mathrm{~d} 3 / 2)$ and $368.0 \mathrm{eV}(\mathrm{Ag}$ $3 \mathrm{~d} 5 / 2$ ), confirming that the small particles deposited after immersion in $\mathrm{AgNO}_{3}$ are metallic Ag. In addition, sulfur 2p electrons were detected only in Ag-NAC-2 and Ag-NAC-5, indicating successful coating.

As shown in Fig. 3, TNS are hydrophilic, with a contact angle $\sim 7^{\circ}$. Notably, the contact angle significantly increased to $56^{\circ}$ after doping with Ag. However, subsequent coating with 2 mM NAC dramatically

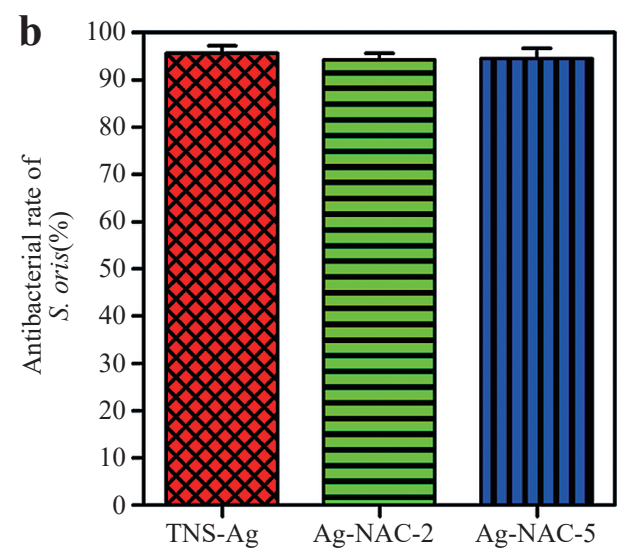

Figure 4. Antibacterial activity of various Ag-doped titanium with nanonetwork structures (TNS-Ag). TNS-Ag and Ag-doped titanium with nanonetwork structures coated with $2 \mathrm{mM}$ (Ag-NAC-2) and $5 \mathrm{mM} \mathrm{N}$-acetyl cysteine (Ag-NAC-5) and tested against S. aureus and A.oris. (a) Antibacterial rate of S.aureus and (b) Antibacterial rate of A.oris. Verticle axis: Antibacterial rate. Antibacterial rate $=(\mathrm{A}-\mathrm{B}) / \mathrm{A} \times 100 \%, \mathrm{~A}$ is the optical density at $600 \mathrm{~nm}$ of bacteria attached on titanium, and B is the optical density at $600 \mathrm{~nm}$ of bacteria attached on Ag-doped titanium and Ag-doped titanium coated with NAC.

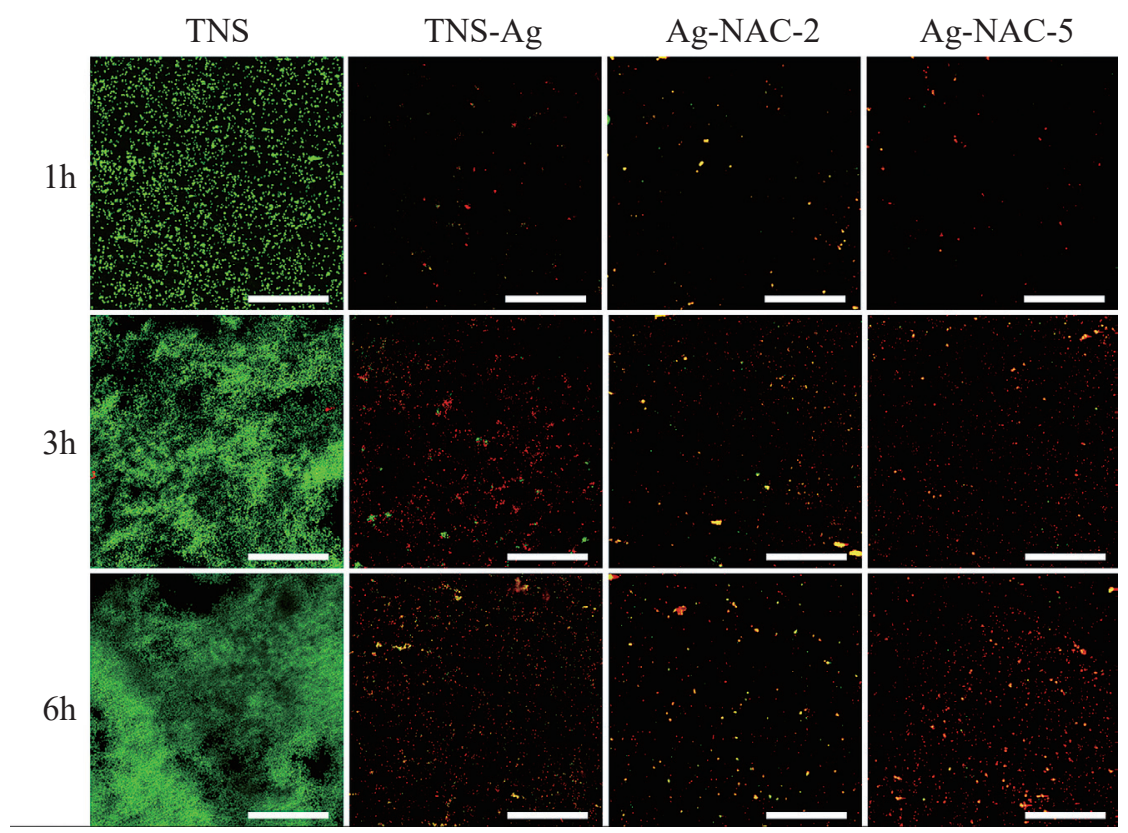

Figure 5. Bacterial viability on various Ag-doped titanium nanonetwork structures. Live (green)/dead (red) staining of $A$. oris attached to titanium with nanonetwork structures (TNS), Ag-doped titanium with nanonetwork structures (TNS-Ag), and Ag-doped titanium titanium with nanonetwork structures coated with 2 $\mathrm{mM}(\mathrm{Ag}-\mathrm{NAC}-2)$ and $5 \mathrm{mM} \mathrm{N}$-acetyl cysteine (Ag-NAC-5). Scale bar $=200 \mu \mathrm{m}$. 


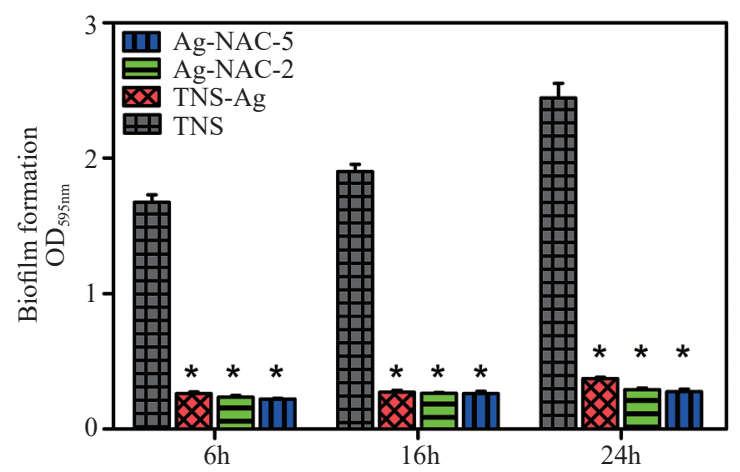

Figure 6. Bacterial biofilm formation on various titanium with nanonetwork structures (TNS). Biofilm formation on TNS, Ag-doped titanium with nanonetwork structures (TNS-Ag), and Ag-doped titanium coated with 2 $\mathrm{mM}(\mathrm{Ag}-\mathrm{NAC}-2)$ and $5 \mathrm{mM} \mathrm{N}$-acetyl cysteine (Ag-NAC-5) was assessed at various time points. $* p<0.05$ vs. titanium with nanonetwork structures (TNS). Verticle axis: optical density at $595 \mathrm{~nm}$ of of bacterial biofilm stained by crystal violet. decreased the surface contact angle to approximately $4^{\circ}$, suggesting that surface hydrophilicity was restored. Notably, materials coated with 5 mM NAC were slightly less hydrophilic than those coated with $2 \mathrm{mM}$ NAC. Since surface hydrophilicity promotes adhesion of bone cells and is a critical indicator of biocompatibility, we anticipated that cells will be recruited more efficiently to the Ag-NAC samples.

\section{Antibacterial activity}

As shown in Fig. 4, the antibacterial activity of S.aureus in $24 \mathrm{~h}$ is $89 \%$ for TNS-Ag, and $87 \%$ and $86 \%$ for Ag-NAC-2 and Ag-NAC-5, respectively. The antibacterial rate of A.oris in $24 \mathrm{~h}$ is slightly higher than S.aureus, $95 \%$ for TNS-Ag, 94\% and 94\% for Ag-NAC-2 and AgNAC-5, respectively. These results indicate that TNS-Ag effectively kills attached bacteria, and that coating with NAC does not compromise this property.

Materials incubated for 1,3 , and $6 \mathrm{~h}$ with $A$. oris were visualized using Live/Dead staining (Fig. 5) to enumerate live (green) and dead (red) a

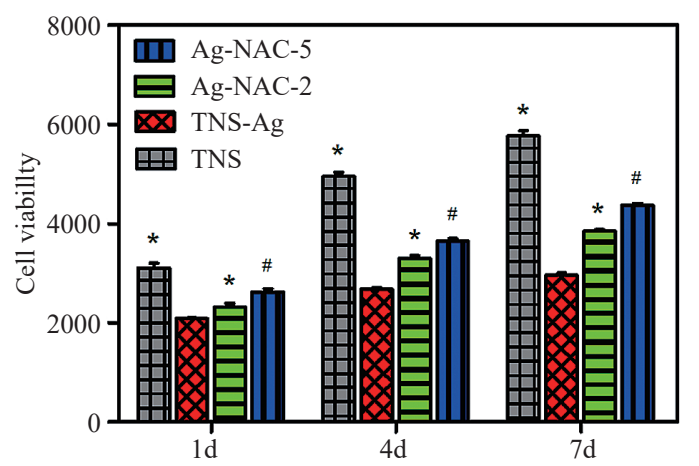

b

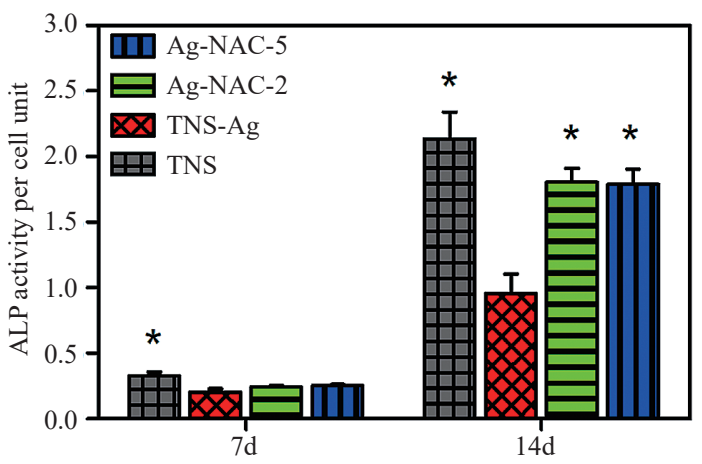

Figure 7. Biocompatibility of various titanium with nanonetwork structures (TNS). (a) Cell viability and (b) alkaline phosphatase activity in rat bone marrow mesenchymal stem cells seeded on TNS, Ag-doped titanium with nanonetwork structures (TNS-Ag), and Ag-doped titanium with nanonetwork structures coated with $2 \mathrm{mM}$ (Ag-NAC-2) and $5 \mathrm{mM} \mathrm{N}$-acetyl cysteine (Ag-NAC-5). ${ }^{*} p<0.05$ vs. Ag-doped titanium with nanonetwork structures (TNS-Ag); ${ }^{*} p<0.05 \mathrm{vs.} \mathrm{Ag-doped}$ titanium with nanonetwork structures coated with $2 \mathrm{mM} \mathrm{N}$-acetyl cysteine (Ag-NAC-2). Veritical axis of a: fluorescence absorbance value of CellTiter-Blue ${ }^{\circledR}$ cell viability assay; veriticle axis of b: fluorescence absorbance value of p-nitrophenol release.

a

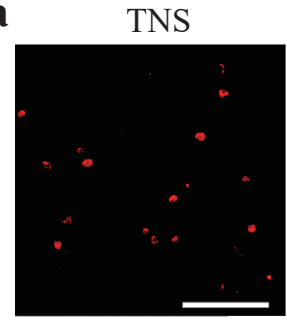

TNS-Ag

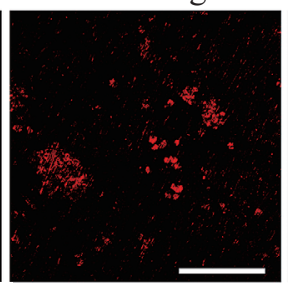

Ag-NAC-2

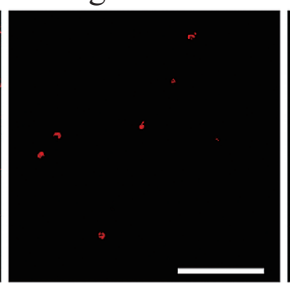

b
Ag-NAC-5

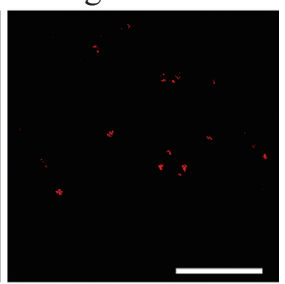

Figure 8. Intracellular ROS in rat bone marrow mesenchymal stem cells seeded on titanium with nanonetwork structures (TNS), Ag-doped titanium with nanonetwork structures (TNS-Ag), and Ag-doped titanium with nanonetwork structures coated with $2 \mathrm{mM}$ (Ag-NAC-2) and 5 mM N-acetyl cysteine (Ag-NAC-5). (a) fluorescent staining images and (b) fluorescence intensity of images that analysed by the embedded software of the confocal laser-scanning microscope. * $p<0.05$ vs. Ag-doped titanium with nanonetwork structures (TNS-Ag); ${ }^{\#} p<0.05$ vs. Ag-doped titanium with nanonetwork structures coated with $2 \mathrm{mM} \mathrm{N}$-acetyl cysteine (Ag-NAC-2). Scale bar $=200 \mu \mathrm{m}$. 
bacteria. TNS were rapidly colonized in $1-6 \mathrm{~h}$ with almost $100 \%$ viable adherent bacteria. However, colonization on TNS-Ag was severely impaired even after $6 \mathrm{~h}$ due to loss of viability of most of the adhered cells. Similar results were obtained on Ag-NAC-2 and Ag-NAC-5. A. oris biofilms were also steadily formed in 6-24 h on TNS, but not on TNS-Ag, Ag-NAC-2, and Ag-NAC-5 (Fig. 6). These results confirm that doping with silver nanoparticles effectively inhibits bacterial attachment, proliferation, and biofilm formation, and that coating with NAC does not compromise antibacterial activity.

\section{Biocompatibility}

Based on the viability of seeded rBMMSCs after 1,4 , and $7 \mathrm{~d}$, TNS$\mathrm{Ag}$ is clearly cytotoxic and poorly biocompatible (Fig. 7a). In contrast, Ag-NAC samples were more biocompatible, with cell viability increasing for $7 \mathrm{~d}$. In particular, cell viability was better on Ag-NAC-5 than on Ag-NAC-2. In agreement with these results, the expression of alkaline phosphatase, a marker of osteoconductivity and early osteogenesis, was lowest after 7-14 d on cells seeded on TNS-Ag (Fig. 7b), but was induced at $14 \mathrm{~d}$ in cells seeded on Ag-NAC samples. Collectively, these results indicate that NAC effectively reduces the cytotoxicity of TNSAg. In addition, intracellular ROS, as quantified using a novel probe that fluoresces only upon oxidation (Fig. 8), accumulated within $3 \mathrm{~d}$ in cells seeded on TNS-Ag, compared to cells seeded on TNS. Importantly, ROS accumulation was lower on cells seeded on Ag-NAC-2 and AgNAC-5, compared to cells seeded on TNS. Indeed, the loss of intracellular ROS was more pronounced in cells seeded on Ag-NAC-5, suggesting that NAC inhibits Ag-induced ROS production in rBMMSCs.

\section{Discussion}

The antibacterial mechanism of Ag-NP- doped titanium is still being investigated. A recent study demonstrated that the antibacterial effect of Ag-NP-doped titanium relies on electron-transfer-based ROS production $^{10)}$. Electron transfer occurs between Ag-NPs and the titanium base substrate, which produces extracellular ROS and elevates oxidative stress at the cell-titanium interface, as well as intracellular ROS, which disrupts bacterial respiration and causes physiological changes, resulting in bacterial death. However, as an antioxidant coating used in the present study, NAC does not protect the bacteria from ROS attack induced by TNS-Ag. We speculate that this interesting result may be related to a certain degree of antibacterial activity of NAC. NAC has been demonstrated to be a non-antibiotic compound that possesses antimicrobial activity towards endodontic pathogens and effectively decreases bacterial biofilm formation ${ }^{24,25)}$. Microbial biofilms are bacterial communities with high cell densities in a matrix of hydrated extracellular polymeric substances, which are mostly composed of polysaccharides ${ }^{26,27)}$. NAC can reduce the production of extracellular polysaccharides, thereby disrupting biofilm maturation and reducing bacterial attachment ${ }^{28,29)}$. Another possible reason for the antibacterial activity of NAC is the intracellular overproduction of reduced glutathione. NAC is a precursor of reduced glutathione ${ }^{30)}$. Actinomycetes do not synthesize GSH but produce mycothiol (MSH) as a major thiol ${ }^{31)}$. GSH is toxic to Actinomycetes because the presence of high concentrations of intracellular GSH may result in redox imbalance in Actinomycetes, which contains an alternative thiol for regulating redox activity ${ }^{32)}$. Notably, TNS doped with Ag and coated with NAC is slightly less toxic against $S$. aureus, which uses glutathione to preserve the intracellular redox balance. In any case, the results clearly show that $\mathrm{Ag}$-doped titanium and $\mathrm{Ag}$-doped titanium coated with NAC possess strong antibacterial activity against $S$. aureus and $A$. oris.
The results of biocompatibility analysis demonstrated that the cytotoxicity of TNS-Ag was effectively improved by NAC coating through suppressing intracellular ROS production. Previous research has confirmed that oxidative stress is a critical mechanism of Ag-induced cytotoxicity ${ }^{33)}$. Indeed, excess ROS may not only damage mitochondria, but also exacerbate ROS formation since mitochondria are the principal source of intracellular ROS in most mammalian cells ${ }^{34)}$. In addition, Ag nanoparticles were shown to induce DNA damage and cell apoptosis through membrane peroxidation, ROS, and oxidative stress ${ }^{35,36)}$. Therefore, reducing oxidative stress may significantly improve the biocompatibility of Ag nanoparticles, and hence, NAC was used in this study as an antioxidant. Following cell uptake, NAC is rapidly deacetylated into cysteine and eventually converted to glutathione, a potent cytoprotectant that removes intracellular and extracellular $\operatorname{ROS}^{37,38)}$. Thus, NAC protects cells from oxidative stress by increasing antioxidant defense capacity $^{39)}$ and direct scavenging activity ${ }^{40,41)}$. Moreover, NAC was reported to promote osteogenesis in titanium implants ${ }^{42,43)}$ by enhancing differentiation of bone marrow stromal cells into osteoblast precursor cells and eventually into secretory osteoblasts ${ }^{44)}$, further highlighting its value in implants. However, extensive studies are required to reveal the mechanism underlying inhibition of ROS production by Ag nanoparticles on titanium implant surface, and in vivo experiments are warranted for verifying the anti-ROS overproduction effect of NAC-coated Agdoped TNS.

In conclusion, titanium with nanonetwork structures were sequentially doped with Ag nanoparticles to enhance antibacterial activity and then coated with NAC to improve biocompatibility. Compared to titanium with nanonetwork structures, Ag-doped titanium with nanonetwork structures exhibited significantly decreased hydrophilicity, which was, however, restored by coating with NAC. Ag-doped titanium with nanonetwork structures was also strongly antibacterial and cytotoxic to a certain extent for rat bone marrow mesenchymal stem cells. Coating with NAC did not compromise the antibacterial activity, but effectively improved biocompatibility by suppressing intracellular ROS production. Thus, NAC coating may be of clinical use in Ag-doped titanium implants with nanonetwork structures.

\section{Acknowledgments}

The authors thank Chiho Mashimo of Osaka University for suggesting test bacteria and for other helpful suggestions. We are also grateful for advice and assistance from members of the Department of Removable Prosthodontics and Occlusion and the Department of Periodontology. This work was supported by grants from the Japan Society for Promotion of Science (16K20524), Science and Technology Program of Guangzhou, China (201804010419), and Science and Technology Program of Shenzhen, China (JCYJ20160428142231354).

\section{Conflicts of Interest}

The authors declare no conflict of interest. The funding sponsor had no role in the design of the study, collection, analyses, or interpretation of data, writing of the manuscript, and decision to publish the results.

\section{References}

1. Lin X, Yang S, Lai K, Yang H, Webster TJ and Yang L. Orthopedic implant biomaterials with both osteogenic and anti-infection capacities and associated in vivo evaluation methods. Nanomedicine 13: 123-142, 2017

2. Goodman SB, Yao Z, Keeney M and Yang F. The future of biologic coatings for orthopaedic implants. Biomaterials 34: 3174-3183, 
Honghao Zhang et al.: Antibacterial Activity and Biocompatibility of Titanium doped with Ag and NAC

2013

3. Jia Z, Xiu P, Li M, Xu X, Shi Y, Cheng Y, Wei S, Zheng Y, Xi T, Cai $\mathrm{H}$ and Liu Z. Bioinspired anchoring AgNPs onto micro-nanoporous $\mathrm{TiO} 2$ orthopedic coatings: Trap-killing of bacteria, surface-regulated osteoblast functions and host responses. Biomaterials 75: 203222,2016

4. Hu H, Zhang W, Qiao Y, Jiang X, Liu X and Ding C. Antibacterial activity and increased bone marrow stem cell functions of $\mathrm{Zn}$-incorporated TiO2 coatings on titanium. Acta Biomater 8: 904-915, 2012

5. Jin G, Qin H, Cao H, Qian S, Zhao Y, Peng X, Zhang X, Liu X and Chu PK. Synergistic effects of dual $\mathrm{Zn} / \mathrm{Ag}$ ion implantation in osteogenic activity and antibacterial ability of titanium. Biomaterials 35: 7699-7713, 2014

6. Zhao Y, Cao H, Qin H, Cheng T, Qian S, Cheng M, Peng X, Wang J, Zhang Y, Jin G, Zhang X, Liu X and Chu PK. Balancing the osteogenic and antibacterial properties of titanium by codoping of $\mathrm{Mg}$ and Ag: an in vitro and in vivo study. ACS Appl. Mater Interfaces 7: 17826-17836, 2015

7. Sondi I and Salopek-Sondi B. Silver nanoparticles as antimicrobial agent: a case study on E. coli as a model for Gram-negative bacteria. J Colloid Interface Sci 275: 177-182, 2004

8. Morones JR, Elechiguerra JL, Camacho A, Holt K, Kouri JB, Ramírez JT and Yacaman MJ. The bactericidal effect of silver nanoparticles. Nanotechnology 16: 2346-2353, 2016

9. Shrivastava S, Bera T, Roy A, Singh G, Ramachandrarao P and Dash D. Characterization of enhanced antibacterial effects of novel silver nanoparticles. Nanotechnology 18: 225103, 2007

10. Wang G, Jin W, Qasim AM, Gao A, Peng X, Li W, Feng H and Chu PK. Antibacterial effects of titanium embedded with silver nanoparticles based on electron-transfer-induced reactive oxygen species. Biomaterials 124: 25-34, 2017

11. Yamada M, Ishihara K, Ogawa $T$ and Sakurai K. The inhibition of infection by wound pathogens on scaffold in tissue-forming process using N-acetyl cysteine. Biomaterials 32: 8474-8485, 2011

12. Blasi F, Page C, Rossolini GM, Pallecchi L, Matera MG, Rogliani P and Cazzola M. The effect of $\mathrm{N}$-acetylcysteine on biofilms: implications for the treatment of respiratory tract infections. Respir. Med. 117: 190-197, 2016

13. Rasmussen K, Nikrad J, Reilly C, Li Y and Jones RS. N-Acetyl-1cysteine effects on multi-species oral biofilm formation and bacterial ecology. Lett Appl Microbiol 62: 30-38, 2016

14. Dinicola S, De Grazia S, Carlomagno G and Pintucci JP. N-acetylcysteine as powerful molecule to destroy bacterial biofilms. A systematic review. Eur Rev Med Pharmacol Sci 18: 2942-2948, 2014

15. Palaniswamy U, Lakkam SR, Arya S and Aravelli S. Effectiveness of $\mathrm{N}$-acetyl cysteine, $2 \%$ chlorhexidine, and their combination as intracanal medicaments on Enterococcus faecalis biofilm. J Conserv Dent 19: 17-20, 2016

16. Eroshenko D, Polyudova T and Korobov V. N-acetylcysteine inhibits growth, adhesion and biofilm formation of Gram-positive skin pathogens. Microb Pathog 105: 145-152, 2017

17. Hamed S, Emara M, Shawky RM, El-Domany RA and Youssef T. Silver nanoparticles: antimicrobial activity, cytotoxicity, and synergism with N-acetyl cysteine. J Basic Microbiol 57: 659-668, 2017

18. Xing H, Komasa S, Taguchi Y, Sekino T and Okazaki J. Osteogenic activity of titanium surfaces with nanonetwork structures. Int J Nanomedicine 9: 1741-1755, 2014

19. Kusumoto T, Yin D, Zhang H, Chen L, Nishizaki H, Komasa Y, Okazaki J and Komasa S. Evaluation of the osteointegration of a novel alkali-treated implant system in vivo. J Hard Tissue Biol 26: 355-360, 2017

20. Zhang H, Komasa S, Mashimo C, Sekino T and Okazaki J. Effect of ultraviolet treatment on bacterial attachment and osteogenic activity to alkali-treated titanium with nanonetwork structures. Int J Nanomedicine 12: 4633-4646, 2017

21. Charalampakis G, Leonhardt $\AA$, Rabe P and Dahlén G. Clinical and microbiological characteristics of peri-implantitis cases: a retrospective multicentre study. Clin Oral Implants Res 23: 1045-1054, 2012

22. Rosan B and Lamont RJ. Dental plaque formation. Microbes Infect 2: $1599-1607,2000$

23. Vielkind P, Jentsch H, Eschrich K, Rodloff AC and Stingu CS. Prevalence of Actinomyces spp. in patients with chronic periodontitis. Int J Med Microbiol 305: 682-688, 2015

24. Choi YS, Kim C, Moon JH and Lee JY. Removal and killing of multispecies endodontic biofilms by $\mathrm{N}$-acetylcysteine. Braz J Microbiol 49: 184-188, 2018

25. Moon JH, Choi YS, Lee HW, Heo JS, Chang SW and Lee JY. Antibacterial effects of $\mathrm{N}$-acetylcysteine against endodontic pathogens. $\mathrm{J}$ Microbiol 54: 322-329, 2016

26. del Prado G, Ruiz V, Naves P, Rodríguez-Cerrato V, Soriano F and del Carmen Ponte M. Biofilm formation by Streptococcus pneumoniae strains and effects of human serum albumin, ibuprofen, $\mathrm{N}$-acetyl-1-cysteine, amoxicillin, erythromycin, and levofloxacin. Diagn Microbiol Infect Dis 67: 311-318, 2010

27. Hall-Stoodley L, Costerton JW and Stoodley P. Bacterial biofilms: from the natural environment to infectious diseases. Nat Rev Microbiol 2: 95-108, 2004

28. Jachlewski, S, Jachlewski WD, Linne U, Bräsen C, Wingender J and Siebers B. Isolation of extracellular polymeric substances from biofilms of the thermoacidophilic archaeon Sulfolobus acidocaldarius. Front Bioeng Biotechnol 3: 123, 2013

29. Silveira LF, Baca P, Arias-Moliz MT, Rodríguez-Archilla A and Ferrer-Luque CM. Antimicrobial activity of alexidine alone and associated with $\mathrm{N}$-acetylcysteine against Enterococcus faecalis biofilm. Int J Oral Sci 5: 146-149, 2013

30. Sadowska AM. N-Acetylcysteine mucolysis in the management of chronic obstructive pulmonary disease. Ther Adv Respir Dis 6: 127135,2012

31. Newton GL, Arnold K, Price MS, Sherrill C, Delcardayre SB, Aharonowitz Y, Cohen G, Davies J, Fahey RC and Davis C. Distribution of thiols in microorganisms: mycothiol is a major thiol in most actinomycetes. J Bacteriol 178: 1990-1995, 1996

32. Green RM, Seth A and Connell ND. A peptide permease mutant of Mycobacterium bovis BCG resistant to the toxic peptides glutathione and S-nitrosoglutathione. Infect Immun 68: 429-436, 2000

33. Carlson C, Hussain SM, Schrand AM, Braydich-Stolle LK, Hess KL, Jones RL and Schlager JJ. Unique cellular interaction of silver nanoparticles: size-dependent generation of reactive oxygen species. J Phys Chem B 112: 13608-13619, 2008

34. Murphy MP. How mitochondria produce reactive oxygen species. Biochem J 417: 1-13, 2009

35. Lim DH, Jang J, Kim S, Kang T, Lee K and Choi IH. The effects of sub-lethal concentrations of silver nanoparticles on inflammatory and stress genes in human macrophages using cDNA microarray analysis. Biomaterials 33: 4690-4699, 2012

36. Ahamed M, Alsalhi MS and Siddiqui MK. Silver nanoparticle applications and human health. Clin Chim.Acta 411: 1841-1848, 2010

37. Lean JM, Davies JT, Fuller K, Jagger CJ, Kirstein B, Partington 
GA, Urry ZL and Chambers TJ. A crucial role for thiol antioxidants in estrogen-deficiency bone loss. J Clin Invest 112: 915-923, 2003

38. Stanislawski L, Lefeuvre M, Bourd K, Soheili-Majd E, Goldberg M and Périanin A. TEGDMA-induced toxicity in human fibroblasts is associated with early and drastic glutathione depletion with subsequent production of oxygen reactive species. J Biomed Mater Res A 66: 476-482, 2003

39. Tsukimura N, Yamada M, Aita H, Hori N, Yoshino F, Chang-Il LM, Kimoto K, Jewett A and Ogawa T. N-acetyl cysteine (NAC)-mediated detoxification and functionalization of poly(methyl methacrylate) bone cement. Biomaterials 30: 3378-3389, 2009

40. Yamada $\mathrm{M}$ and Ogawa T. Chemodynamics underlying $\mathrm{N}$-acetyl cysteine-mediated bone cement monomer detoxification. Acta Biomater 5: 2963-2973. 2009

41. Paranjpe A, Sung EC, Cacalano NA, Hume WR and Jewett A.
$\mathrm{N}$-acetyl cysteine protects pulp cells from resin toxins in vivo. $\mathrm{J}$ Dent Res 87: 537-541, 2008

42. Lee YH, Bhattarai G, Park IS, Kim GR, Kim GE, Lee MH and Yi HK. Bone regeneration around $\mathrm{N}$-acetyl cysteine-loaded nanotube titanium dental implant in rat mandible. Biomaterials 34: 1019910208, 2013

43. Suzuki T, Kubo K, Hori N, Yamada M, Kojima N, Sugita Y, Maeda $\mathrm{H}$ and Ogawa $\mathrm{T}$. Nonvolatile buffer coating of titanium to prevent its biological aging and for drug delivery. Biomaterials 31: 48184828, 2010

44. Yamada M, Tsukimura N, Ikeda T, Sugita Y, Att W, Kojima N, Kubo $\mathrm{K}$, Ueno T, Sakurai K and Ogawa T. N-acetyl cysteine as an osteogenesis-enhancing molecule for bone regeneration. Biomaterials 34 : 6147-6156, 2013 